\title{
A Comprehensive Analysis of Nuclear-Encoded Mitochondrial Genes in Schizophrenia
}

Goncalves, Vanessa F.; Cappi, Carolina; Hagen, Christian M.; Sequeira, Adolfo; Vawter, Marquis P.; Derkach, Andriy; Zai, Clement C.; Hedley, Paula L.; Bybjerg-Grauholm, Jonas; Pouget, Jennie G.; Cuperfain, Ari B.; Sullivan, Patrick F.; Christiansen, Michael; Kennedy, James L.; Sun, Lei

Published in:

Biological Psychiatry

DOI:

10.1016/j.biopsych.2018.02.1175

Publication date:

2018

Document version

Publisher's PDF, also known as Version of record

Document license:

CC BY-NC-ND

Citation for published version (APA):

Goncalves, V. F., Cappi, C., Hagen, C. M., Sequeira, A., Vawter, M. P., Derkach, A., Zai, C. C., Hedley, P. L., Bybjerg-Grauholm, J., Pouget, J. G., Cuperfain, A. B., Sullivan, P. F., Christiansen, M., Kennedy, J. L., \& Sun, L. (2018). A Comprehensive Analysis of Nuclear-Encoded Mitochondrial Genes in Schizophrenia. Biological

Psychiatry, 83(9), 780-789. https://doi.org/10.1016/j.biopsych.2018.02.1175 


\title{
A Comprehensive Analysis of Nuclear-Encoded Mitochondrial Genes in Schizophrenia
}

\author{
Vanessa F. Gonçalves, Carolina Cappi, Christian M. Hagen, Adolfo Sequeira, Marquis P. Vawter, \\ Andriy Derkach, Clement C. Zai, Paula L. Hedley, Jonas Bybjerg-Grauholm, Jennie G. Pouget, \\ Ari B. Cuperfain, Patrick F. Sullivan, Michael Christiansen, James L. Kennedy, and Lei Sun
}

\begin{abstract}
BACKGROUND: The genetic risk factors of schizophrenia (SCZ), a severe psychiatric disorder, are not yet fully understood. Multiple lines of evidence suggest that mitochondrial dysfunction may play a role in SCZ, but comprehensive association studies are lacking. We hypothesized that variants in nuclear-encoded mitochondrial genes influence susceptibility to SCZ.

METHODS: We conducted gene-based and gene-set analyses using summary association results from the Psychiatric Genomics Consortium Schizophrenia Phase 2 (PGC-SCZ2) genome-wide association study comprising 35,476 cases and 46,839 control subjects. We applied the MAGMA method to three sets of nuclear-encoded mitochondrial genes: oxidative phosphorylation genes, other nuclear-encoded mitochondrial genes, and genes involved in nucleus-mitochondria crosstalk. Furthermore, we conducted a replication study using the iPSYCH SCZ sample of 2290 cases and 21,621 control subjects.

RESULTS: In the PGC-SCZ2 sample, 1186 mitochondrial genes were analyzed, among which 159 had $p$ values $<.05$ and 19 remained significant after multiple testing correction. A meta-analysis of 818 genes combining the PGC-SCZ2 and iPSYCH samples resulted in 104 nominally significant and nine significant genes, suggesting a polygenic model for the nuclear-encoded mitochondrial genes. Gene-set analysis, however, did not show significant results. In an in silico protein-protein interaction network analysis, 14 mitochondrial genes interacted directly with 158 SCZ risk genes identified in PGC-SCZ2 (permutation $p=.02$ ), and aldosterone signaling in epithelial cells and mitochondrial dysfunction pathways appeared to be overrepresented in this network of mitochondrial and SCZ risk genes.

CONCLUSIONS: This study provides evidence that specific aspects of mitochondrial function may play a role in SCZ, but we did not observe its broad involvement even using a large sample.
\end{abstract}

Keywords: Gene-gene interaction, GWAS-HD, MAGMA, Mitochondria, Oxidative phosphorylation, Schizophrenia, Stratified FDR

https://doi.org/10.1016/j.biopsych.2018.02.1175

Schizophrenia (SCZ) is a severe psychiatric disorder for which the underlying causes are far from fully understood. The brain is highly dependent on oxidative metabolism as the primary source of energy to maintain its functions (1), and a disturbance in brain energy metabolism has been suggested as part of the pathogenesis of SCZ (2). Studies have indicated that abnormalities in glucose oxidative metabolism may contribute to an individual's susceptibility of developing psychosis $(1,3)$. Indeed, there is compelling evidence that patients with SCZ have impaired glucose metabolism, with decreased metabolic rates and reduced blood flow in the frontal cortex exhibited during cognitive tasks (4-7).

Impaired oxidative metabolism suggests mitochondrial dysfunction mainly because these organelles are the primary sources of aerobic energy via oxidative phosphorylation (OXPHOS) for neuronal function. Furthermore, it has been shown that mitochondria play an important role in cellular processes that are disturbed in SCZ, such as synaptic transmission (8-10) and oxidative stress (11). Comorbidity between mitochondrial diseases and neuropsychiatric symptomatology has also been reported $(12,13)$.

SCZ has a strong genetic basis, with estimated heritability around $80 \%$ (14). However, few studies aim specifically to discover mitochondrial loci associated with SCZ, and consequently, genetic data supporting the association of mitochondrial loci with SCZ are lacking. There is some evidence that mitochondrial genes play a role in SCZ [for a review, see Hjelm et al. (9)]. For example, the most recent results from the Psychiatric Genomics Consortium Schizophrenia Phase 2 (PGC-SCZ2) genome-wide association study (GWAS) $(35,476$ cases and 46,839 control subjects), the largest dataset to date used for genetic association studies of SCZ, reported evidence for 22 nuclear-encoded mitochondrial genes (15), but mitochondria-related pathways showed no significant association with the disease in another large study of SCZ (9379 cases and 7736 control subjects) (16). Further evidence 
includes enrichment of expression quantitative trait loci (17), and an excess of large $(>500 \mathrm{~Kb})$ deletions, and rare copy number variants in nuclear-encoded mitochondrial genes in individuals with SCZ (18).

Here we tested the hypothesis that variants in nuclearencoded mitochondrial genes influence SCZ risk. Because mitochondrial genes have unique features (being encoded by both the nuclear and mitochondrial genomes) and contain highly connected signaling pathways (for maintenance of mitochondria homeostasis), we performed both gene-based and gene-set/pathway analyses.

Of particular interest is the set of nuclear-encoded OXPHOS genes, the mitochondrial pathway that produces adenosine triphosphate (ATP) for neuronal functioning. This specific pathway has been more intensively investigated in SCZ, mainly due to the critical role of mitochondrial reactive oxygen species in triggering oxidative stress. SCZ patients have been reported to show increased levels of oxidative stress (2), and the SCZoxidative stress association has been noted in functional studies (19). Moreover, there is compelling evidence linking reactive oxygen species with immune-inflammatory pathways $(20,21)$, which have also been implicated in $\operatorname{SCZ}(22,23)$.

Specific nuclear-encoded OXPHOS genes physically interact with other subunits encoded by the mitochondrial DNA. Therefore, the mitochondria and nucleus must coordinate transcription, translation, import, and function of mitochondrial protein complexes (24). Disturbances in this crosstalk may lead to deficiencies in the mitochondrial ATP production process, causing or contributing to disease.

We defined three sets of genes to test our hypothesis that variants in mitochondrial or mitochondria-related genes influence SCZ risk: 1) nuclear-encoded OXPHOS genes, 2) other nuclear-encoded mitochondrial genes (excluding the OXPHOS genes), and 3) genes involved in the crosstalk between the nucleus and mitochondria (25). Using the MAGMA tool (26) alongside a hypothesis-driven GWAS (GWAS-HD) approach (27), we aimed to 1) identify and replicate new mitochondrial susceptibility loci for SCZ, 2) evaluate the significance of the three mitochondrial gene sets in SCZ, and 3) use in silico tools to predict the biological processes involving mitochondrial genes identified in this study, to potentially reveal novel abnormal cellular circuits in SCZ.

\section{METHODS AND MATERIALS}

\section{Samples}

We used the summary association statistics available from the most recent PGC-SCZ2 GWAS (15) to perform our analyses in the discovery sample. The original quality control steps for 35,476 SCZ cases and 46,839 control subjects from 52 cohorts, and detailed association analyses of 9,444,230 single nucleotide polymorphisms (SNPs) are described elsewhere (15).

To replicate findings, we used the iPSYCH GWAS data (www.ipsych.au.dk) with 2290 SCZ cases and 21,621 control subjects. Subjects were genotyped using the Illumina Infinium PsychChip v1.0 (Illumina, Inc., San Diego, CA) in accordance with the manufacturer's instructions. The total number of genotyped SNPs was 588,454 (28). Preimputation quality control steps included removal of SNPs with minor allele frequency $<0.05$ and Hardy-Weinberg equilibrium $p<10^{-6}$ in control subjects, and removal of individuals with call rate $<95 \%$ and (cryptic) first- and second-degree relatives using KING (v1.9) (29). The data were then phased using Shapeit3 (v2.r837) (30) and imputed using IMPUTE2 (v2.3.2) (31), with the 1000 Genomes Project Phase 3 as the reference panel. Postimputation quality control included removal of SNPs with INFO score $<0.2$, minor allele frequency $<0.001$, genotype missing rate $>10 \%$, and Hardy-Weinberg equilibrium $p<$ $10^{-6}$, resulting in a total of $8,842,045$ SNPs for analysis.

\section{Nuclear-Encoded Mitochondrial Genes and Mitochondria-Related Gene Sets}

The OXPHOS gene set started with 95 nuclear-encoded genes selected using KEGG (32). All 95 were listed in the MitoCarta 2.0 release 2015 (33), a database of human genes with strong evidence for mitochondrial location based on computational and microscopy evidence. In total, 89 genes tagged by 6686 PGC-SCZ2 GWAS SNPs formed the OXPHOS gene set.

The mitochondrial gene set originally included 1158 nuclear-encoded genes downloaded from MitoCarta. Two genes encoded in the extended major histocompatibility complex (34) were excluded; the major histocompatibility complex region is known to be highly associated with $\mathrm{SCZ}$ and was thus excluded for a conservative analysis. After removal of these genes, 1038 genes tagged by 131,502 SNPs remained, and they formed the mitochondrial gene set (excluding the OXPHOS genes defined by the gene set above).

The nucleus-mitochondria crosstalk gene set included 76 genes selected based on previous literature (24,35-37). These genes were tagged by 18,781 SNPs. One gene also appeared in the OXPHOS group, and 16 genes appeared in the mitochondrial group. We decided to keep these overlapping genes in both groups because they may be part of several different cellular processes/circuits involving mitochondria.

In total, 155,843 unique SNPs were located within the genomic region of 1186 unique mitochondrial or mitochondriarelated genes; only SNPs within transcribed regions were included to minimize overlapping of SNPs between multiple genes and inclusion of null SNPs (38).

\section{MAGMA Gene-Based and Gene Set Analyses}

The PGC-SCZ2 GWAS $p$ values (after the genomic control [GC] adjustment) were the primary input data for MAGMA (v1.06) with the 1000 Genomes Project Phase 3 (Build 37/European data only) used as the reference panel. We calculated the linkage disequilibrium score intercept for the PGC-SCZ2 GWAS data and found a value of 1.052 , suggesting a slight inflation even after correction for the polygenic nature of SCZ. Thus, we applied the conservative GC correction to the original SNP association results before conducting our analyses, ensuring that the remaining enrichment was genuinely due to a polygenic model.

The MAGMA gene-based results (using the SNP-wise option) were then corrected for multiple testing of the 1186 mitochondrial genes using the Bonferroni method. MAGMA gene-set competitive analysis [with default parameter values and correcting for potential confounders and linkage disequilibrium score between genes (26)] was then performed to examine if any of the three mitochondrial gene sets was 
enriched in variants associated with SCZ (three hypotheses tested).

\section{Hypothesis-Driven GWAS}

Given that the MAGMA method only analyzed the defined genes and gene sets, we used the GWAS-HD and stratified false discovery rate (sFDR) (27) to complement the MAGMA analysis. GWAS-HD re-evaluates (upweight or downweight through the sFDR control) all individual SNP associations genome wide, based on the prior information contained in the biological hypothesis. SNPs annotated to the three mitochondrial gene sets were assigned to three high-priority strata, and the remaining SNPs formed a separate (low priority) stratum. We emphasize here that SNP stratification must be done a priori independent of the $p$ values to be stratified. The sFDR method improves power if the prior information is informative; the high-priority group(s) indeed contains proportionally more truly associated SNPs as compared with no stratification (39).

\section{Replication Study}

To replicate association between individual SNPs and SCZ, a list of 657 SNPs (with sFDR $q$ value $<.05$ ) were sent to the iPSYCH Mitochondria Group investigators in Denmark. The same MAGMA approach used in the discovery PGC-SCZ2 sample was applied to the iPSYCH SCZ sample to replicate the genebased findings. A meta-analysis using Fisher's method to combine the discovery and replication gene-based $p$ values was performed. Genes with $p$ values less than the conservative Bonferroni correction threshold were declared significant.

\section{Mitochondrial Gene Expression in the Brain}

We used the Allen Human Brain Atlas to explore the expression of the associated genes across human brain regions to look for additional evidence of their potential role in SCZ. Microarray data for 6 subjects ( 4 men and 2 women 18 to 68 years of age with no known neuropsychiatric or neuropathological history) were downloaded from Allen Human Brain Atlas (http://human.brain-map.org/static/download) in July 2017. The Atlas used a custom design Agilent $8 \times 60 \mathrm{~K}$ array that included the $4 \times 44 \mathrm{~K}$ Agilent Whole Human Genome probe set supplemented with an additional 16,000 probes. As probes for the same gene were highly correlated, only one probe per gene was selected to avoid collinearity. This probe selection was based on expression levels, with moderate expression levels defined conservatively as $>6$; the median expression for all genes across the brain for the 6 subjects was 4.99 and the 75th quartile was 7.13 .

\section{Differential Expression of Genes in SCZ}

The .bam files were obtained through the CommonMind Consortium Knowledge Portal (https://www.synapse.org/ $\mathrm{CMC}$ ) (the largest RNA sequencing dataset for human dorsolateral prefrontal cortex) with permission and analyzed at University of California, Irvine (40). Data from 10 duplicate samples and 89 individuals with incomplete demographic information were excluded. The analysis included 514 samples and the following factors were used to correct for differences between brain samples: $\mathrm{pH}$, age, postmortem interval, RNA integrity index, batch, institution, and the manner of death.
The linear regression model included the following terms in R package (glm) (R Project for Statistical Computing, Vienna, Austria): Gene Expression (log counts per million) $=\beta 0+\beta 1 \times$ (diagnosis) $+\beta 2 \times($ brain $\mathrm{pH})+\beta 3 \times$ (postmortem interval) + $\beta 4 \times($ age $)+\beta 5 \times($ sex $)+\beta 6 \times(\mathrm{RIN})+\beta 7 \times$ (institution) + $\beta 8 \times($ cause of death $)+)+\varepsilon$.

Log counts per million values were obtained from RNA sequencing read counts divided by the corresponding library size (in millions) to yields counts per million (41). Postmortem interval is in hours, RNA integrity number was obtained from Agilent BioAnalyzer, and institution was coded for each of three sites (Icahn School of Medicine at Mount Sinai, University of Pittsburgh, University of Pennsylvania).

\section{Gene-Gene Interaction and Overrepresentation Analyses}

Genes that belong to the same molecular networks and cellular pathways are often involved in the pathophysiology of the same or similar conditions (41). Therefore, we mapped functionally relevant physical interactions using an in silico protein-protein interaction (PPI) network analysis. We tested gene-gene interactions among genes that code for interacting proteins, particularly the 19 nuclear-encoded mitochondrial genes significantly associated with SCZ in the discovery sample using a gene-based approach, as well as the 327 genes in the 108 genome-wide significant loci reported in the original PGC-SCZ2 study (15).

We first built a PPI network with the 327 SCZ risk genes (of which 10 were not recognized by the program for encoding microRNAs) to visualize how these genes interact among each other in a functional network using GeneMANIA Cytoscape Plugin (Cytoscape_v3.5.1) (42). These initial 327 genes were referred as seed genes. To visualize and analyze topological parameters of the network, we used the Cytoscape platform (43) and the CentiScape plugin (44). After constructing the network, we then used GeneNets toolbox in MATLAB (R2015a; The MathWorks, Inc., Natick, MA) (45) to determine whether the connectivity among the 327 seed genes was statistically significant compared with a randomly selected network of similar size (taking into account number of genes and genes sizes).

We then used GeneNets to investigate whether this network formed by 327 seed genes (the SCZ risk genes) significantly connect with the backbone genes (i.e., mitochondrial genes considered significant in this study), using the default seed randomization option. We called the network built from this analysis the mito-SCZ network. Mitochondrial and SCZ genes physically interacting in the mito-SCZ network were selected for gene-set overrepresentation analysis in the GeNets software (Broad Institute of MIT and Harvard, Cambridge, MA).

GeNets is a web platform (http://apps.broadinstitute.org/ genets) for analysis and interpretation of genetic variation in the context of gene-gene networks (46). GeNets allowed us to visualize genes that interact with each other as well as their communities (i.e., a group of genes relatively more connected with each other in a network), while taking into account the biological process related to the genes. Additional candidate genes could be predicted as well. This software investigates 
which pathways within the gene set of interest are enriched, using a Bonferroni-adjusted hypergeometric test. In our study, only gene sets from the Molecular Signatures Database were used.

GeNets also includes Quack (https://www.biorxiv.org/ content/biorxiv/early/2017/10/04/196303.full.pdf), a machine learning algorithm that can be trained to find nontrivial pathway relationships between genes of interest. The hypothesis is that genes in a common pathway would share pathway-specific topological properties (connectivity profiles) that systematically distinguish them from genes that are not part of the pathway under analysis. The algorithm generates a confidence score (from 0 to 1) that ranks each gene; genes with higher scores have greater probability being related to the pathway under analysis.

Last, we used Ingenuity Pathway Analysis software (v33559992, release date March 28, 2017; QIAGEN Bioinformatics, Redwood City, CA) with default parameter values to determine whether the genes in the mito-SCZ network were enriched for specific biological pathways. The $p$ values were adjusted using the default option, i.e., the FDR-adjusted $p$ (Benjamini-Hochberg method) (47). We used the lenient FDR control to identify as many candidate pathways as possible while still maintaining an acceptable false positive rate.

\section{RESULTS}

\section{Gene-Based and Gene-Set Analysis}

We used MAGMA to conduct gene-based analysis of the 1186 OXPHOS, mitochondrial, and nucleus-mitochondria crosstalk genes using PGC-SCZ2 as our discovery sample. In total, 159 mitochondrial genes had MAGMA gene-based $p<.05,19$ of which survived Bonferroni correction (Table 1). In total, 818 mitochondrial genes had data in both the PGC-SCZ2 discovery and IPSYCH replication samples, and meta-analysis resulted in 104 genes with gene-based $p$ $<.05$ and nine with $p<.05 / 818$ (Bonferroni correction) (Table 2). Notably, among 1000 randomly selected sets of 818 genes from the nuclear genome, the numbers of genebased $p<.05$ ranged from 66 to 123, with a mean of 92.15 (first quartile of 84 , median of 92.5 , and third quartile of 98). Among the total of 1000 replicates, there were 46 replicates with counts $\geq 104$ (the value observed in the set of nuclear encoded mitochondrial genes), resulting in an empirical $p=.046$ for the enrichment analysis of truly associated genes. This finding suggests that the polygenic model known for the nuclear genome appears to hold for the set of nuclear-encoded mitochondrial genes analyzed. Furthermore, q-q plots, expressed on a -log10(p) scale in Figure 1, show evidence for enrichment, with an estimated proportion of associated genes of $3 \%, 8 \%$, and $13 \%$ for the discovery, replication, and combined samples, respectively. These estimates are subject to sampling variation (48). We also note that the $q-q$ plot for a set of $p$ values with a higher estimated proportion of truly associated variants (e.g., Figure 1B for the replication sample) could look more "flat" than Figure 1A for the discovery sample, if the corresponding statistical effect sizes (which depends on both genetic effect size of a variant and the sample size) differ.

With respect to gene-set analysis, none of the three sets of genes showed statistically significant enrichment of association based on MAGMA, with gene-set $p$ values equal to .40 , .64 , and .19 for the OXPHOS, mitochondrial excluding

Table 1. Mitochondrial Genes Significantly Associated With SCZ Risk at the $p<.05$ Level After the Conservative Bonferroni Correction for 1186 Multiple Hypothesis Tests

\begin{tabular}{|c|c|c|c|c|c|c|c|c|c|}
\hline Symbol & $\mathrm{CHR}$ & Start & Stop & $\begin{array}{l}\text { No. of SNPs } \\
\text { Annotated }\end{array}$ & $\begin{array}{l}\text { No. of Relevant } \\
\text { Parameters Used } \\
\text { in the Model }\end{array}$ & $n$ & ZSTAT & $p$ Value & Correct_p $p^{a}$ \\
\hline APOPT1 & 14 & 104029299 & 104057236 & 59 & 17 & 82,315 & 5.9449 & 1.38E-09 & .000002 \\
\hline C12orf65 & 12 & 123717844 & 123742651 & 68 & 11 & 82,315 & 5.8275 & 2.81E-09 & .000003 \\
\hline HSPE1-MOB4 & 2 & 198364721 & 198418423 & 63 & 16 & 82,315 & 5.5313 & $1.59 \mathrm{E}-08$ & .000019 \\
\hline C2orf69 & 2 & 200775979 & 200792996 & 43 & 13 & 82,315 & 5.5011 & 1.89E-08 & .000022 \\
\hline C2orf47 & 2 & 200820040 & 200828848 & 19 & 10 & 82,315 & 4.8352 & $6.65 \mathrm{E}-07$ & .0008 \\
\hline PCCB & 3 & 135969167 & 136056737 & 148 & 43 & 82,315 & 4.8174 & 7.27E-07 & .0009 \\
\hline ATPAF2 & 17 & 17921334 & 17942483 & 25 & 11 & 82,315 & 4.7175 & 1.19E-06 & .0014 \\
\hline HSPA9 & 5 & 137890571 & 137911318 & 37 & 16 & 82,315 & 4.5898 & $2.22 \mathrm{E}-06$ & .0026 \\
\hline DNAJA3 & 16 & 4475806 & 4506776 & 71 & 29 & 82,315 & 4.3822 & 5.87E-06 & .0070 \\
\hline NT5DC2 & 3 & 52558385 & 52569093 & 26 & 8 & 82,315 & 4.34 & 7.12E-06 & .0084 \\
\hline LETM2 & 8 & 38240041 & 38267045 & 36 & 13 & 82,315 & 4.3314 & 7.41E-06 & .0088 \\
\hline NDUFA6 & 22 & 42481530 & 42486888 & 9 & 5 & 82,315 & 4.3137 & 8.03E-06 & .0095 \\
\hline HSPD1 & 2 & 198351308 & 198364998 & 18 & 7 & 82,315 & 4.2994 & 8.56E-06 & .01 \\
\hline DNAJC19 & 3 & 180701497 & 180707562 & 4 & 4 & 82,315 & 4.267 & 9.91E-06 & .01 \\
\hline COQ10B & 2 & 198318231 & 198339851 & 20 & 6 & 82,315 & 4.1766 & $1.48 \mathrm{E}-05$ & .02 \\
\hline SMDT1 & 22 & 42475695 & 42480288 & 5 & 3 & 82,315 & 4.1677 & $1.54 \mathrm{E}-05$ & .02 \\
\hline FOXO3 & 6 & 108881026 & 109005972 & 171 & 32 & 82,315 & 4.1328 & 1.79E-05 & .02 \\
\hline HSPE1 & 2 & 198364721 & 198368187 & 2 & 2 & 82,315 & 4.0746 & 2.3E-05 & .03 \\
\hline$A B C B 9$ & 12 & 123405498 & 123451056 & 42 & 18 & 82,315 & 4.0223 & 2.88E-05 & .03 \\
\hline
\end{tabular}

CHR, chromosome; SCZ, schizophrenia; SNPs, single nucleotide polymorphisms; ZSTAT, $Z$ value for the gene.

${ }^{a} p$ values after the conservative Bonferroni correction. 
Table 2. List of Nine Mitochondrial Genes Significantly Associated With SCZ Risk Through Meta-analysis at the $p<.05$ Level After the Conservative Bonferroni Correction for $\mathbf{8 1 8}$ Multiple Hypothesis Tests

\begin{tabular}{|c|c|c|c|c|c|c|c|c|c|c|c|}
\hline \multirow[b]{2}{*}{ Symbol } & \multirow[b]{2}{*}{$\mathrm{CHR}$} & \multirow[b]{2}{*}{ Start } & \multirow[b]{2}{*}{ Stop } & \multicolumn{3}{|c|}{ Discovery } & \multicolumn{3}{|c|}{ Replication } & \multicolumn{2}{|c|}{ Meta-analysis } \\
\hline & & & & $n$ & ZSTAT & disc_p $p$ & $n$ & ZSTAT & rep_p & Fisher_Combined_p & Correct_p $p^{a}$ \\
\hline HSPE1-MOB4 & 2 & 198364721 & 198418423 & 82,315 & 5.5313 & $1.59 \mathrm{E}-08$ & 23,911 & -0.683 & .7527 & $2.30 \mathrm{E}-07$ & .0002 \\
\hline C2orf47 & 2 & 200820040 & 200828848 & 82,315 & 4.8352 & $6.65 \mathrm{E}-07$ & 23,911 & 0.55423 & .2897 & $3.17 \mathrm{E}-06$ & .0026 \\
\hline SMDT1 & 22 & 42475695 & 42480288 & 82,315 & 4.1677 & $1.54 \mathrm{E}-05$ & 23,911 & 2.0424 & .0206 & $5.05 \mathrm{E}-06$ & .0041 \\
\hline ATPAF2 & 17 & 17921334 & 17942483 & 82,315 & 4.7175 & $1.19 \mathrm{E}-06$ & 23,911 & 0.49159 & .3115 & $5.88 \mathrm{E}-06$ & .0048 \\
\hline PCCB & 3 & 135969167 & 136056737 & 82,315 & 4.8174 & $7.27 \mathrm{E}-07$ & 23,911 & -1.4194 & .9221 & $1.02 \mathrm{E}-05$ & .0083 \\
\hline NDUFA6 & 22 & 42481530 & 42486888 & 82,315 & 4.3137 & 8.03E-06 & 23,911 & 1.2234 & .1106 & 1.33E-05 & .0108 \\
\hline HSPA9 & 5 & 137890571 & 137911318 & 82,315 & 4.5898 & $2.22 \mathrm{E}-06$ & 23,911 & -0.24631 & .5973 & 1.93E-05 & .0158 \\
\hline DNAJA3 & 16 & 4475806 & 4506776 & 82,315 & 4.3822 & $5.87 \mathrm{E}-06$ & 23,911 & 0.35949 & .3596 & 2.97E-05 & .0243 \\
\hline FOXO3 & 6 & 108881026 & 109005972 & 82,315 & 4.1328 & $1.79 \mathrm{E}-05$ & 23,911 & 0.69366 & .2440 & 5.83E-05 & .0477 \\
\hline
\end{tabular}

CHR, chromosome code; disc, discovery sample; rep, replication sample; SCZ, schizophrenia; ZSTAT, $Z$ value for the gene.

${ }^{a} p$ values after the conservative Bonferroni correction.

OXPHOS, and nucleus-mitochondria crosstalk gene sets, respectively. This result could be a true negative, although genetic heterogeneity may also drive these nonsignificant findings.

\section{GWAS-HD Analysis}

In total, summary statistics of 9,444,230 PGC-SCZ2 GWAS SNPs were available, among which 155,843 unique SNPs were located within the genomic region of 1186 mitochondrial genes as defined above. GC-corrected $p$ values were then used for the GWAS-HD analysis. Specifically, FDR control was simultaneously applied to the four strata of SNPs: stratum 1, containing 6686 OXPHOS SNPs; stratum 2, containing 131,502 mitochondrial SNPs; stratum 3, containing 18,781 nucleusmitochondria crosstalk SNPs (1126 overlap with stratum 2); and stratum 4, containing the remaining 9,239,959 GWAS SNPs, excluding the major histocompatibility complex region as discussed before.

In total, 728 mitochondrial SNPs had an sFDR $q$ value $<.05$, including 86 SNPs previously reported as genome-wide significant $\left[p<5 \times 10^{-8}(49)\right]$ (Supplemental Table S1). The GWAS-HD significant SNPs mapped to 54 genes, of which all had MAGMA gene-based $p<.05$, including the 19 genes declared significant in the PGC-SCZ2 discovery sample using our approach.

Of the 728 mitochondrial SNPs, 657 with rs numbers (indels variants excluded) were used for replication analysis using the independent iPSYCH SCZ sample. In total, 40 SNPs had meta-analysis $p$ value $<.05$, 35 of which demonstrated an effect in the same direction as in the PGCSCZ2 discovery sample (Table 3). These 35 SNPs were located within five genes (APOPT1, NDUFA6, SMDT1, MSRA, and $C A M K K 2$ ), all of which showed at least nominally significant MAGMA gene-based association with SCZ in our discovery sample.

\section{Mitochondrial Gene Expression in the Brain}

All 19 mitochondrial genes associated with SCZ in this study were expressed at significant levels in human brain tissue (Supplemental Figure S1), and 17 showed particularly high
A

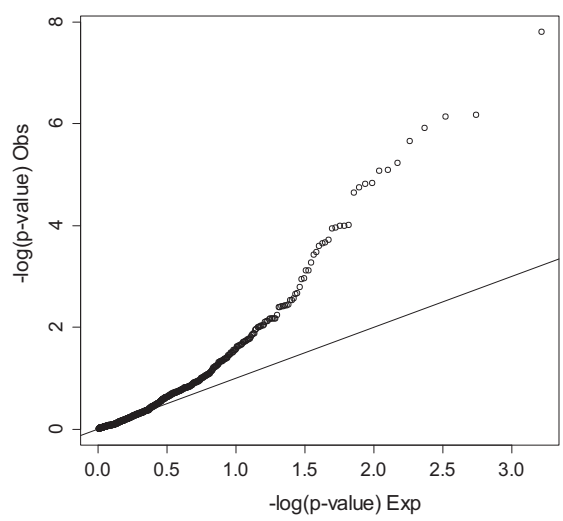

Discovery Sample $\pi_{1}=3 \%$
B

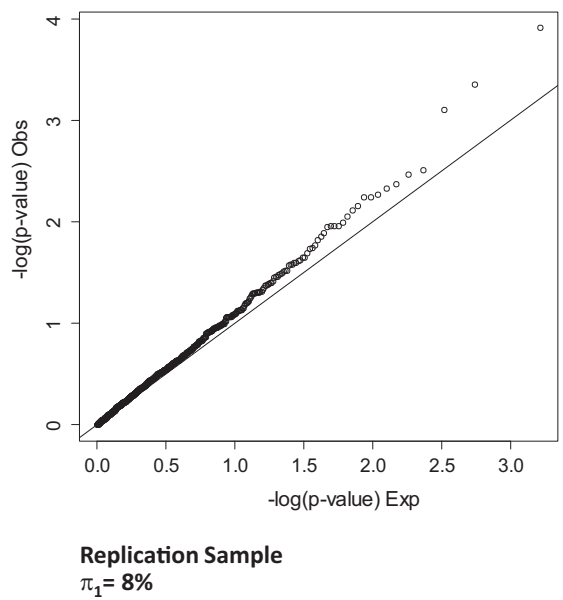

C

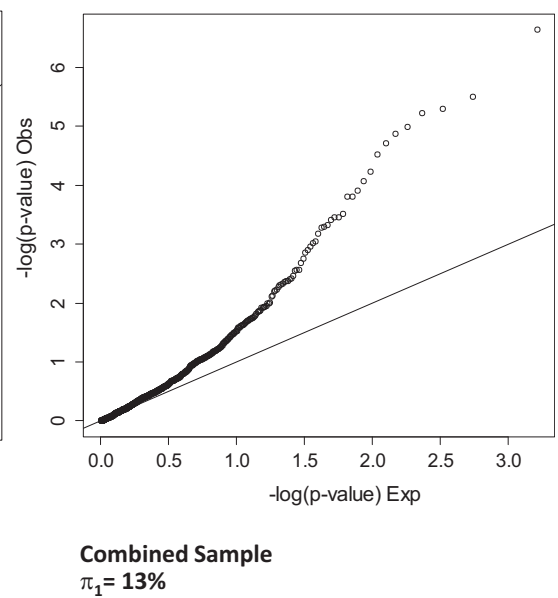

Figure 1. The q-q plot for the (A) discovery, (B) replication, (C) and combined discovery and replication samples. The estimated proportion of associated genes $(\pi)$ is shown. Exp, expected; Obs, observed. 
Table 3. List of 35 SNPs Replicated in the iPSYCH SCZ Sample

\begin{tabular}{|c|c|c|c|c|c|c|c|c|c|c|c|c|c|c|}
\hline \multirow[b]{2}{*}{ SNP } & \multirow[b]{2}{*}{$\mathrm{CHR}$} & \multirow[b]{2}{*}{ POS } & \multirow[b]{2}{*}{ A1 } & \multirow[b]{2}{*}{$\mathrm{A} 2$} & \multicolumn{4}{|c|}{ Discovery } & \multicolumn{4}{|c|}{ Replication } & \multicolumn{2}{|c|}{ Meta-analysis } \\
\hline & & & & & $n$ & OR & SE & $p$ Value & $n$ & OR & SE & $p$ Value & meta_P & corrected_meta_P \\
\hline rs10431750 & 14 & 104040065 & $\mathrm{~T}$ & C & 82,315 & - & 0.0115 & $2.11 \mathrm{E}-09$ & 23,371 & - & 0.03401 & .03 & $2.67 \mathrm{E}-10$ & 9.35E-09 \\
\hline rs1063843 & 12 & 121681687 & $\mathrm{~T}$ & C & 82,315 & + & 0.0131 & $4.37 \mathrm{E}-04$ & 23,911 & + & 0.03833 & .02 & $2.26 \mathrm{E}-05$ & 7.92E-04 \\
\hline rs12887734 & 14 & 104046834 & $T$ & $\mathrm{G}$ & 82,315 & + & 0.0117 & $7.03 \mathrm{E}-10$ & 23,229 & + & 0.03413 & .03 & $1.06 \mathrm{E}-10$ & 3.70E-09 \\
\hline rs12889403 & 14 & 104034746 & $\mathrm{~T}$ & C & 82,315 & + & 0.0116 & 7.06E-09 & 23,562 & + & 0.034 & .04 & 1.25E-09 & $4.38 \mathrm{E}-08$ \\
\hline rs12889721 & 14 & 104034863 & $T$ & C & 82,315 & + & 0.0116 & $9.54 \mathrm{E}-09$ & 23,575 & + & 0.03399 & .05 & $2.06 \mathrm{E}-09$ & $7.20 \mathrm{E}-08$ \\
\hline rs12890820 & 14 & 104034849 & $T$ & $\mathrm{C}$ & 82,315 & - & 0.0116 & $5.71 \mathrm{E}-09$ & 23,584 & - & 0.03399 & .05 & 1.19E-09 & 4.17E-08 \\
\hline rs12894729 & 14 & 104035292 & A & $G$ & 82,315 & + & 0.0116 & 8.18E-09 & 23,556 & + & 0.03401 & .04 & 1.47E-09 & $5.13 \mathrm{E}-08$ \\
\hline rs1653591 & 12 & 121681138 & A & $G$ & 82,315 & + & 0.0131 & $1.69 \mathrm{E}-04$ & 23,840 & + & 0.03844 & .02 & $9.59 \mathrm{E}-06$ & 3.35E-04 \\
\hline rs2071711 & 22 & 42477650 & $C$ & $G$ & 82,315 & - & 0.0114 & 3.55E-05 & 23,842 & - & 0.03409 & .02 & 1.92E-06 & $6.72 \mathrm{E}-05$ \\
\hline rs2269524 & 22 & 42475703 & $\mathrm{~T}$ & $G$ & 82,315 & + & 0.0113 & $2.75 \mathrm{E}-05$ & 23,845 & + & 0.03404 & .02 & 1.61E-06 & 5.64E-05 \\
\hline rs2274267 & 14 & 104029449 & A & $G$ & 82,315 & + & 0.0117 & $1.14 \mathrm{E}-08$ & 23,418 & + & 0.03404 & .03 & 1.35E-09 & 4.71E-08 \\
\hline rs2296482 & 14 & 104029819 & $\mathrm{~T}$ & $\mathrm{G}$ & 82,315 & - & 0.0116 & 7.01E-09 & 23,708 & - & 0.03387 & .02 & $6.78 \mathrm{E}-10$ & $2.37 \mathrm{E}-08$ \\
\hline rs2403197 & 14 & 104053764 & $\mathrm{~T}$ & $C$ & 82,315 & + & 0.0115 & 1.89E-09 & 23,205 & + & 0.03424 & .05 & 4.27E-10 & 1.49E-08 \\
\hline rs2668247 & 12 & 121684176 & $C$ & $G$ & 82,315 & - & 0.0131 & $1.69 \mathrm{E}-04$ & 23,819 & - & 0.03848 & .02 & 1.02E-05 & 3.57E-04 \\
\hline rs2686340 & 12 & 121682051 & $\mathrm{~T}$ & $C$ & 82,315 & + & 0.0131 & $1.81 \mathrm{E}-04$ & 23,742 & + & 0.03855 & .02 & 9.45E-06 & 3.31E-04 \\
\hline rs2686342 & 12 & 121685188 & A & $T$ & 82,315 & - & 0.0131 & $2.17 \mathrm{E}-04$ & 23,785 & - & 0.03846 & .02 & $1.34 \mathrm{E}-05$ & 4.70E-04 \\
\hline rs2686344 & 12 & 121690548 & $\mathrm{~T}$ & C & 82,315 & + & 0.0125 & 4.96E-04 & 22,506 & + & 0.03729 & .02 & 2.95E-05 & $1.03 E-03$ \\
\hline rs2686345 & 12 & 121690555 & $\mathrm{~T}$ & $\mathrm{C}$ & 82,315 & + & 0.0125 & $5.01 \mathrm{E}-04$ & 22,506 & + & 0.03729 & .02 & 2.99E-05 & $1.04 \mathrm{E}-03$ \\
\hline rs34026011 & 14 & 104050883 & $A$ & $G$ & 82,315 & + & 0.0115 & 1.12E-09 & 23,205 & + & 0.03418 & .03 & $1.90 \mathrm{E}-10$ & 6.66E-09 \\
\hline rs35026580 & 14 & 104042753 & A & $G$ & 82,315 & - & 0.0115 & 1.96E-09 & 23,316 & - & 0.03405 & .02 & 1.93E-10 & $6.74 \mathrm{E}-09$ \\
\hline rs35229468 & 14 & 104052057 & $\mathrm{~T}$ & C & 82,315 & + & 0.0115 & 1.97E-09 & 23,212 & + & 0.03421 & .04 & $3.45 \mathrm{E}-10$ & $1.21 \mathrm{E}-08$ \\
\hline rs35496194 & 14 & 104031351 & $\mathrm{~T}$ & C & 82,315 & + & 0.0117 & $2.66 \mathrm{E}-09$ & 23,651 & + & 0.03395 & .04 & $5.42 \mathrm{E}-10$ & $1.90 \mathrm{E}-08$ \\
\hline rs4147640 & 22 & 42485292 & $\mathrm{~T}$ & $\mathrm{C}$ & 82,315 & + & 0.0113 & $1.94 \mathrm{E}-05$ & 23,850 & + & 0.03405 & .02 & 1.23E-06 & 4.29E-05 \\
\hline rs4147641 & 22 & 42482502 & $C$ & $G$ & 82,315 & + & 0.0113 & $2.43 \mathrm{E}-05$ & 23,846 & + & 0.03405 & .02 & 1.56E-06 & 5.47E-05 \\
\hline rs4906336 & 14 & 104033265 & $C$ & $G$ & 82,315 & - & 0.0116 & 5.17E-09 & 23,558 & - & 0.034 & .05 & 1.13E-09 & 3.94E-08 \\
\hline rs4906337 & 14 & 104040414 & A & C & 82,315 & + & 0.0115 & $2.12 \mathrm{E}-09$ & 23,349 & + & 0.03403 & .02 & $2.13 \mathrm{E}-10$ & 7.47E-09 \\
\hline rs 4906338 & 14 & 104050357 & A & $\mathrm{G}$ & 82,315 & - & 0.0115 & $1.25 \mathrm{E}-09$ & 23,173 & - & 0.03419 & .03 & $1.90 \mathrm{E}-10$ & $6.66 \mathrm{E}-09$ \\
\hline rs58033365 & 14 & 104030901 & $T$ & $\mathrm{C}$ & 82,315 & - & 0.0116 & $6.50 \mathrm{E}-09$ & 23,711 & - & 0.03389 & .03 & $8.01 \mathrm{E}-10$ & $2.80 \mathrm{E}-08$ \\
\hline rs 6002592 & 22 & 42476754 & A & C & 82,315 & + & 0.0113 & $3.46 \mathrm{E}-05$ & 23,844 & + & 0.03404 & .02 & $2.04 \mathrm{E}-06$ & 7.13E-05 \\
\hline rs6002596 & 22 & 42484466 & $A$ & $G$ & 82,315 & - & 0.0114 & 1.36E-05 & 23,846 & - & 0.03406 & .02 & 8.94E-07 & 3.13E-05 \\
\hline rs7140568 & 14 & 104041374 & $\mathrm{~T}$ & C & 82,315 & + & 0.0115 & $2.12 \mathrm{E}-09$ & 23,335 & + & 0.03404 & .02 & $1.97 \mathrm{E}-10$ & $6.88 \mathrm{E}-09$ \\
\hline rs71417868 & 14 & 104046592 & A & $T$ & 82,315 & - & 0.0115 & 1.32E-09 & 23,237 & - & 0.03411 & .03 & $1.83 \mathrm{E}-10$ & $6.40 \mathrm{E}-09$ \\
\hline rs73191548 & 8 & 10035997 & A & G & 82,315 & + & 0.0109 & $6.21 \mathrm{E}-05$ & 23,451 & + & 0.03213 & .04 & 6.73E-06 & $2.36 \mathrm{E}-04$ \\
\hline rs8017628 & 14 & 104047428 & $T$ & C & 82,315 & + & 0.0115 & 1.44E-09 & 23,233 & + & 0.03413 & .03 & $2.24 \mathrm{E}-10$ & 7.85E-09 \\
\hline rs8017993 & 14 & 104047734 & $A$ & $G$ & 82,315 & - & 0.0115 & 1.69E-09 & 23,217 & - & 0.03417 & .03 & $2.78 \mathrm{E}-10$ & 9.73E-09 \\
\hline
\end{tabular}

A1, allele 1; A2, allele 2; CHR, chromosome code; OR, odds ratio; POS, chromosome location; SCZ, schizophrenia; SE, standard error; SNP, single nucleotide polymorphism.

levels of expression in the thalamic, midbrain, and striatal areas. Importantly, these brain regions are known to be altered in SCZ (50). For 17 of these 19 genes, we observed moderateto-high levels of log2 expression (log2 score $>6$ ) across the human brain (Supplemental Table S2). The two other genes had moderate to high expression levels in specific areas such as the frontal lobe for $A B C B 9$ or the cerebellar cortex for LETM2.

\section{Differential Gene Expression of Replicated Genes in SCZ}

Of the nine replicated genes shown in Table 2, three were downregulated in a nominally significant manner in SCZ compared with control subjects (Table 4).

\section{PPI Network and Gene Set Overrepresentation Analysis}

The PPI analysis of the 337 SCZ risk genes showed a connected network including 327 seed genes and 5790 interactions (edges in Supplemental Figure S2) between them. The randomization method showed that the connectivity among 327 genes was statistically significant $(p=.034,1000$ randomizations). The greatest number of interactions between genes in the network was found for those with coexpression, (i.e., two genes showing similar expression levels in the Gene Expression Omnibus database, suggestive of coexpression) (Supplemental Figure S2).

In examining the interaction between the 19 mitochondrial genes (backbone genes) with the 327 SCZ risk genes 
Table 4. Fold Change of Replicated Mitochondria Genes in the Dorsolateral Prefrontal Cortex From the CommonMind Consortium

\begin{tabular}{lcccccc}
\hline & $\begin{array}{c}\text { Average } \\
\text { Expression } \\
\text { (CPM) }\end{array}$ & $t$ & $\begin{array}{c}p \\
\text { Value }\end{array}$ & $\begin{array}{c}\text { Fold Change } \\
\text { (Schizophrenia/Control } \\
\text { Symbol }\end{array}$ & FDR $^{a}$ \\
\hline NDUFA6 & 5.91 & -2.13 & .03 & -1.21 & 0.25 \\
\hline PCCB & 5.78 & -2.73 & .01 & -1.19 & 0.14 \\
\hline SMDT1 & 4.28 & -2.86 & .00 & -1.23 & 0.12 \\
\hline
\end{tabular}

Three mitochondria genes shown in Table 2 were nominally significantly decreased in the expression for schizophrenia compared with control subjects for the CommonMind Consortium dorsolateral prefrontal cortex after correction for brain $\mathrm{pH}$, age, postmortem interval, RNA integrity number, batch, institution, and the manner of death.

CPM, counts per million; FDR, false discovery rate.

${ }^{a}$ Benjamini-Hochberg-adjusted $p$ value.

(seed genes), we observed that 14 mitochondrial genes were connected with 158 SCZ genes in a significant network after the randomization test $(p=.023,1000$ randomizations) (Figure 2 and Supplemental Figure S3). The genes DNAJC19, HSPA9, PCCB, and HSPE1 showed the greatest number of connections among mitochondrial genes, interacting with many genes of the SCZ network.

For the GeNets analysis of functional connectivity among the 14 mitochondrial genes and 158 SCZ genes, 70 additional candidates were included to the network, based on their connectivity with our input genes (selected by the software). Of the 242 genes examined, 138 genes were connected in a significant network $(p=.002)$, of which 18 were mitochondrial. Moreover, mitochondrial genes comprised one of the largest communities in the network (Community 7; Supplemental Figure S4). For this community, two significant canonical pathways were overrepresented: 1) respiratory electron transport and 2) ATP synthesis. Furthermore, the gene NDUFA6 from our study was one of the genes included in this community (Supplemental Table S4). Additionally, GeNets classified the mitochondrial genes DRG2, NDUFA2, NDUFA6, and NDUFA13 as the most important genes for the network based on Quack (Supplemental Table S5).

Finally, the Ingenuity Pathway Analysis of the 14 mitochondrial genes and the 158 SCZ genes (mito-SCZ network) found canonical biological pathways related to mitochondrial function as overrepresented in this network (after FDR correction). Particularly, mitochondrial dysfunction and aldosterone signaling in epithelial cells were most overrepresented (Supplemental Table S6).

\section{DISCUSSION}

Regions of the genome robustly associated with schizophrenia by the PGC-SCZ2 GWAS provide a framework for secondary analysis to identify important biological pathways and help to shed further light on disease etiology. Although there were 22 mitochondrial genes among the top PGC-SCZ2 GWAS hits, the mitochondrial system is not yet widely considered as a pathophysiologic mechanism in SCZ. To the best of our knowledge, the study presented here is the first to examine explicitly the role of mitochondria-related genes in SCZ.

Using MAGMA gene-based analysis, we identified 19 mitochondrial or mitochondria-related genes significantly associated with SCZ at gene level, all of which were corroborated in a separate GWAS-HD sFDR analysis. Of the 19 significant genes identified in the PGC-SCZ2 sample, 12 were from the mitochondrial gene set, five were from the nucleusmitochondria-crosstalk gene set, and two were from the OXPHOS gene set. We note that the significance here is
A

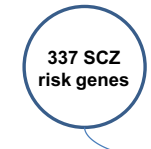

Ripke et al., Nature, 2014

\section{SCZ network} (327 genes)
B

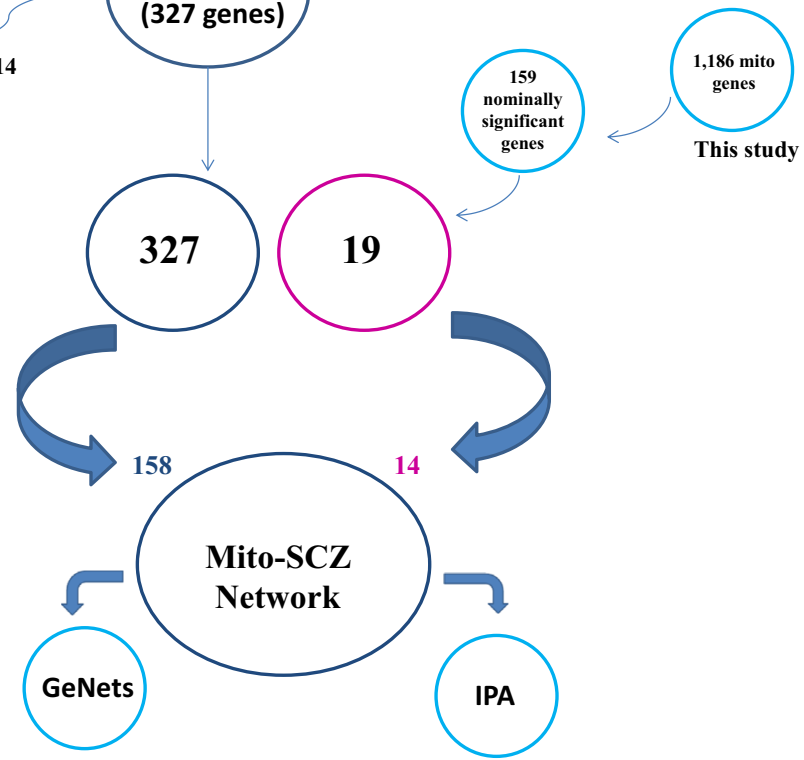

Figure 2. Protein-protein interaction analysis workflow. (A) Protein-protein interaction analysis of 337 schizophrenia (SCZ) risk genes (15) showed a significant connected network including 327 of these genes $(p=.03)$. (B) These 327 genes were further investigated using GeneNets and found to be connected in a network with significant mitochondrial genes from our study $(n=19)$. (C) A total of $158 \mathrm{SCZ}$ risk genes were connected with 14 mitochondrial genes in a significant network $(p=.02)$ (mito-SCZ network). (D) Pathways and biological processes associated with the mito-SCZ network were investigated using GeNets and Ingenuity Pathway Analysis (IPA) software. 
defined based on Bonferroni correction for the 1186 genes tested using MAGMA. However, eight genes overlapped with those declared as genome-wide significant loci by the PGCSCZ2 GWAS; the remaining 11 novel genes only passed the less conservative threshold as defined here. We did not include regulatory regions in our gene-based analysis and it remains to be explored further in the future.

Meta-analysis of 818 genes with data in both the PGCSCZ2 (discovery) and iPSYCH (replication) samples identified 104 genes with meta-analysis $p<.05$, and nine were significant after Bonferroni correction for multiple testing.

The $q-q$ plots of gene-based $p$ values in Figure 1 were suggestive of enrichment of variants associated with SCZ. This is supported by the stratified $q-q$ plots of SNP-based $p$ values in Supplementary Figure S5 where the estimated proportion of associated SNPs was 3\% for the mitochondrial gene set (GC $\lambda=1.04$ ), and $4 \%$ for the nucleus-mitochondria crosstalk gene set $(\lambda=1.10)$, as compared with $0.01 \%$ for the protein-coding control group $(\lambda=0.99)$. The SNPs in the OXPHOS gene set, however, did not show apparent enrichment $(\lambda=0.78,0.00 \%)$.

However, we did not find enrichment for the three mitochondrial gene sets tested when we used the MAGMA pathway analysis tool. One possible explanation is that the mitochondrial system includes many internal pathways that are connected with several distinct cellular circuits. Thus, future studies need to be more refined in terms of defining the mitochondria-internal pathways to be tested.

We then performed a network-based analysis to explore the biological significance of our findings. Our in silico investigations suggested that 14 mitochondrial genes were functionally connected with risk genes for SCZ and identified several biological processes in which mitochondrial genes were involved in SCZ (respiratory electron transport, respiratory electron transport/ATP synthesis, and aldosterone signaling in epithelial cells).

Of interest, HSPA9 is a chaperone involved in protein quality control, folding, and degradation, and it has been associated with Alzheimer's disease (51). The expression of DNAJC19 has also been found to be downregulated in the motor cortex and thalamus of patients with autism (52). The involvement of respiratory electron transport and ATP synthesis pathways support impaired OXPHOS functioning in SCZ subjects, which has already been proposed as part of the etiology of the disease (11).

The aldosterone signaling pathway is involved in the regulation of mineral metabolism. Interestingly, aldosterone is thought to disinhibit $\mathrm{N}$-methyl-D-aspartate receptors due to its role in the excretion of magnesium ions $\left(\mathrm{Mg}^{2+}\right)$, which may reduce intracerebral $\mathrm{Mg}^{2+}$ levels (53). $\mathrm{N}$-methyl-D-aspartate receptor hypofunction might explain (at least in part) common clinical manifestations of SCZ including cognitive deficits, impaired memory and learning, positive-negative symptoms, and executive dysfunction (54).

NDUFA6 was the mitochondrial gene with the highest Quack score (0.104). This gene has been found to be downregulated in bipolar patients and has a central role in energy metabolism and mitochondrial function (55). NDUFA6 is also downregulated in the analysis of CommonMind Consortium dorsolateral prefrontal cortex expression in SCZ compared with control subjects. Another gene, DRG2, is a SCZ risk gene found to be involved in the regulation of mitochondrial morphology and function (56).

Furthermore, we examined whether the mitochondrial genes connect directly with the SCZ genes, which are considered important for disease etiology or treatment (15). We found that DNAJC19, HSPA9, and PCCB had high connectivity with the following SCZ genes: ATP2A2 (calcium signaling), CNTN4 (neuronal network formation and plasticity), and FXR1 (neurodevelopment).

We have also identified four nucleus-mitochondria crosstalk genes listed as associated with SCZ in our gene-based study. These genes are involved with the mitochondrial unfolded protein response. This pathway is activated under mitochondrial stress and induces the expression of protective genes (chaperones and proteases) to re-establish protein homeostasis within mitochondria. It may also result in the maintenance of mitochondria with heteroplasmic deleterious mitochondrial DNA variants (57).

In conclusion, our study was able to identify novel nuclearencoded mitochondrial and mitochondria-related (nucleusmitochondria crosstalk) genes associated with SCZ using a gene-based approach. We did not obtain significant gene-set findings, potentially limited by low signal-to-noise ratio, but we were able to provide supporting evidence for the variants' association from a GWAS-HD analysis of individual SNPs. Our in silico analysis of the top findings suggests that mitochondrial pathways should be further explored in SCZ research. Importantly, our approach can be applied, with relative ease, to other complex diseases with a hypothesized mitochondrial component such as bipolar disorder, Alzheimer's disease, Parkinson's disease, and diabetes.

\section{ACKNOWLEDGMENTS AND DISCLOSURES}

The research reported in this article was supported by the Novo Nordisk Foundation (the iPSYCH study was conducted using the Danish National Biobank resource); the Lundbeck Foundation Initiative for Integrative Psychiatric Research; the Broad at MIT and Harvard - iPSYCH community; Genome Canada (to JLK); the Ontario Ministry of Research and Innovation (to JLK); the Canadian Institutes of Health Research (to JLK and LS); a Brain Behavior and Research Foundation/NARSAD Distinguished Investigator Award (to JLK); the Judy and Larry Tanenbaum Foundation (to JLK and VFG); the Natural Sciences and Engineering Research Council of Canada (to LS); National Institute of Mental Health Grant Nos. R01MH097082 (to AS), R21MH099440-01 (to MPV), and R01MH085801 (to MPV); Fundação de Amparo à Pesquisa do Estado de São Paulo Grant No. 2014/01585-5 (to CC); and Integrated Platform Carlos Chagas CNPq Grant No. 460928/20147 (to $\mathrm{CC}$ ).

We would like to acknowledge the Judy and Larry Tanenbaum Family Foundation for the support to this study. The authors are also grateful to the subjects in the PGC and iPSYCH studies for their participation.

PFS is on the Scientific Advisory Board for Pfizer, Inc. JLK is a Scientific Advisory Board member (unpaid) of AssureRx Health Inc. and has received speaker honoraria and expenses from Eli Lilly, Novartis, and Shire and consultant honoraria and expenses from Roche. All authors report no biomedical financial interests or potential conflicts of interest.

\section{ARTICLE INFORMATION}

From the Department of Psychiatry (VFG, CCZ, JGP, ABC, JLK); Department of Statistical Sciences (AD, LS), Faculty of Arts and Science; and Biostatistics Division (LS), Dalla Lana School of Public Health, University of Toronto; and Neuroscience Section (VFG, CCZ, JGP, ABC, JLK), Centre for Addiction and Mental Health, Toronto, Ontario, Canada; Department of 
Psychiatry (CC), School of Medicine, University of São Paulo, São Paulo, Brazil; Department for Congenital Disorders (CMH, PLH, JB-G, MC), Statens Serum Institut; and Department of Biomedical Science (MC), University of Copenhagen, Copenhagen, Denmark; Department of Psychiatry and Human Behavior (AS, MPV), University of California, Irvine, California; Department of Genetics (PFS), University of North Carolina, Chapel Hill, North Carolina; and the Department of Medical Epidemiology and Biostatistics (PFS), Karolinska Institutet, Stockholm, Sweden.

VFG and CC contributed equally to this work.

Address correspondence to Vanessa F. Gonçalves, Ph.D., 250 College Street, Room 129, 1st Floor. Toronto, Ontario M5T-1R8, Canada; E-mail: Vanessa.Goncalves@camh.ca.

Received Jul 18, 2017; revised Feb 26, 2018; accepted Feb 27, 2018.

Supplementary material cited in this article is available online at https:// doi.org/10.1016/j.biopsych.2018.02.1175.

\section{REFERENCES}

1. Blass JP (2002): Glucose/mitochondria in neurological conditions. Int Rev Neurobiol 51:325-376.

2. Prabakaran S, Swatton JE, Ryan MM, Huffaker SJ, Huang JT, Griffin JL, et al. (2004): Mitochondrial dysfunction in schizophrenia: Evidence for compromised brain metabolism and oxidative stress. Mo Psychiatry 9:684-697, 643.

3. Kooy FH (1919): Hyperglycemia in mental disorders. Brain 42:214-289.

4. Wolkin A, Jaeger J, Brodie JD, Wolf AP, Fowler J, Rotrosen J, et al. (1985): Persistence of cerebral metabolic abnormalities in chronic schizophrenia as determined by positron emission tomography. Am J Psychiatry 142:564-571.

5. Berman KF, Torrey EF, Daniel DG, Weinberger DR (1992): Regional cerebral blood flow in monozygotic twins discordant and concordant for schizophrenia. Arch Gen Psychiatry 49:927-934.

6. Jacobsen LK, Hamburger SD, Van Horn JD, Vaituzis AC, McKenna K, Frazier JA, et al. (1997): Cerebral glucose metabolism in childhood onset schizophrenia. Psychiatry Res 75:131-144.

7. Hill K, Mann L, Laws KR, Stephenson CM, Nimmo-Smith I, McKenna PJ (2004): Hypofrontality in schizophrenia: A meta-analysis of functional imaging studies. Acta Psychiatr Scand 110:243-256.

8. Arion D, Corradi JP, Tang S, Datta D, Boothe F, He A, et al. (2015): Distinctive transcriptome alterations of prefrontal pyramidal neurons in schizophrenia and schizoaffective disorder. Mol Psychiatry 20: 1397-1405.

9. Hjelm BE, Rollins B, Mamdani F, Lauterborn JC, Kirov G, Lynch G, et al. (2015): Evidence of mitochondrial dysfunction within the complex genetic etiology of schizophrenia. Mol Neuropsychiatry 1:201-219.

10. Ly CV, Verstreken P (2006): Mitochondria at the synapse. Neuroscientist 12:291-299.

11. Bitanihirwe BK, Woo TU (2011): Oxidative stress in schizophrenia: An integrated approach. Neurosci Biobehav Rev 35:878-893.

12. Fattal O, Budur K, Vaughan AJ, Franco K (2006): Review of the literature on major mental disorders in adult patients with mitochondrial diseases. Psychosomatics 47:1-7.

13. Anglin RE, Rosebush PI, Noseworthy MD, Tarnopolsky M, Weber AM, Soreni N, et al. (2013): Metabolite measurements in the caudate nucleus, anterior cingulate cortex and hippocampus among patients with mitochondrial disorders: A case-control study using proton magnetic resonance spectroscopy. CMAJ Open 1:E48-E55.

14. Sullivan PF, Kendler KS, Neale MC (2003): Schizophrenia as a complex trait: Evidence from a meta-analysis of twin studies. Arch Gen Psychiatry 60:1187-1192.

15. Ripke SNB, Corvin A, Walters JT, Farh KH, Holmans PA, et al. (2014): Biological insights from 108 schizophrenia-associated genetic loci. Nature 511:421-427.

16. O'Dushlaine CRL, Lee PH, Duncan L, Parikshak NN, Newhouse S, et al. (2015): Psychiatric genome-wide association study analyses implicate neuronal, immune and histone pathways. Nat Neurosci 18:199-209.

17. Kim Y, Xia K, Tao R, Giusti-Rodriguez P, Vladimirov V, van den Oord E, et al. (2014): A meta-analysis of gene expression quantitative trait loci in brain. Transl Psychiatry 4:e459.
18. Szatkiewicz JP, O'Dushlaine C, Chen G, Chambert K, Moran JL, Neale BM, et al. (2014): Copy number variation in schizophrenia in Sweden. Mol Psychiatry 19:762-773.

19. Rajasekaran A, Venkatasubramanian G, Berk M, Debnath M (2014): Mitochondrial dysfunction in schizophrenia: Pathways, mechanisms and implications. Neurosci Biobehav Rev 48:10-21.

20. Pedrini M, Massuda R, Fries GR, de Bittencourt Pasquali MA, Schnorr CE, Moreira JC, et al. (2012): Similarities in serum oxidative stress markers and inflammatory cytokines in patients with overt schizophrenia at early and late stages of chronicity. J Psychiatr Res 46:819-824.

21. Anderson G, Berk M, Dodd S, Bechter K, Altamura AC, Dell'osso B, et al. (2013): Immuno-inflammatory, oxidative and nitrosative stress, and neuroprogressive pathways in the etiology, course and treatment of schizophrenia. Prog Neuropsychopharmacol Biol Psychiatry 42:1-4.

22. Leza JC, Garcia-Bueno B, Bioque M, Arango C, Parellada M, Do K, et al. (2015): Inflammation in schizophrenia: A question of balance. Neurosci Biobehav Rev 55:612-626.

23. Muller N (2016): What role does inflammation play in schizophrenia? Expert Rev Neurother 16:1337-1340.

24. Quiros PM, Mottis A, Auwerx J (2016): Mitonuclear communication in homeostasis and stress. Nat Rev Mol Cell Biol 17:213-226.

25. Woodson JD, Chory J (2008): Coordination of gene expression between organellar and nuclear genomes. Nat Rev Genet 9: 383-395.

26. de Leeuw CA, Mooij JM, Heskes T, Posthuma D (2015): MAGMA: Generalized gene set analysis of GWAS data. PLoS Comput Biol 11:e1004219.

27. Sun L, Rommens JM, Corvol H, Li W, Li X, Chiang TA, et al. (2012): Multiple apical plasma membrane constituents are associated with susceptibility to meconium ileus in individuals with cystic fibrosis. Nat Genet 44:562-569.

28. Pedersen CB, Byberg-Grauholm J, Pedersen MG, Grove J, Agerbo E, Baekvad-Hansen M, et al. (2018): The iPSYCH-2012 case-cohort sample: New directions for unravelling genetic and environmental architectures of severe mental disorders. Mol Psychiatry 23:6-14.

29. Manichaikul A, Mychaleckyj JC, Rich SS, Daly K, Sale M, Chen WM (2010): Robust relationship inference in genome-wide association studies. Bioinformatics 26:2867-2873.

30. O'Connell J, Sharp K, Shrine N, Wain L, Hall I, Tobin M, et al. (2016): Haplotype estimation for biobank-scale data sets. Nat Genet 48 : 817-820.

31. Howie B, Fuchsberger C, Stephens M, Marchini J, Abecasis GR (2012): Fast and accurate genotype imputation in genomewide association studies through pre-phasing. Nat Genet 44:955-959

32. Kanehisa M, Furumichi M, Tanabe M, Sato Y, Morishima K (2017): KEGG: New perspectives on genomes, pathways, diseases and drugs. Nucleic Acids Res 45:D353-D361.

33. Calvo SE, Clauser KR, Mootha VK (2015): MitoCarta2.0: An updated inventory of mammalian mitochondrial proteins. Nucleic Acids Res 44:D1251-D1257.

34. Horton R, Wilming L, Rand V, Lovering RC, Bruford EA, Khodiyar VK, et al. (2004): Gene map of the extended human MHC. Nat Rev Genet 5:889-899.

35. Biswas G, Guha M, Avadhani NG (2005): Mitochondria-to-nucleus stress signaling in mammalian cells: Nature of nuclear gene targets, transcription regulation, and induced resistance to apoptosis. Gene 354:132-139.

36. Pellegrino MW, Nargund AM, Haynes CM (2013): Signaling the mitochondrial unfolded protein response. Biochim Biophys Acta 1833: 410-416.

37. Cagin U, Enriquez JA (2015): The complex crosstalk between mitochondria and the nucleus: What goes in between? Int J Biochem Cel Biol 63:10-15.

38. Rietveld CA, Medland SE, Derringer J, Yang J, Esko T, Martin NW, et al. (2013): GWAS of 126,559 individuals identifies genetic variants associated with educational attainment. Science 340:1467-1471. 
39. Sun L, Craiu RV, Paterson AD, Bull SB (2006): Stratified false discovery control for large-scale hypothesis testing with application to genomewide association studies. Genet Epidemiol 30:519-530.

40. Fromer M, Roussos P, Sieberts SK, Johnson JS, Kavanagh DH, Perumal TM, et al. (2016): Gene expression elucidates functional impact of polygenic risk for schizophrenia. Nat Neurosci 19:1442-1453.

41. Gandhi TK, Zhong J, Mathivanan S, Karthick L, Chandrika KN, Mohan SS, et al. (2006): Analysis of the human protein interactome and comparison with yeast, worm and fly interaction datasets. Nat Genet 38:285-293.

42. Warde-Farley D, Donaldson SL, Comes O, Zuberi K, Badrawi R, Chao P, et al. (2010): The GeneMANIA prediction server: Biological network integration for gene prioritization and predicting gene function. Nucleic Acids Res 38:W214-W220.

43. Franz M, Lopes CT, Huck G, Dong Y, Sumer O, Bader GD (2016): Cytoscape.js: A graph theory library for visualisation and analysis. Bioinformatics 32:309-311.

44. Scardoni G, Petterlini M, Laudanna C (2009): Analyzing biological network parameters with CentiScaPe. Bioinformatics 25:2857-2859.

45. Taylor A, Steinberg J, Andrews TS, Webber C (2015): GeneNet Toolbox for MATLAB: A flexible platform for the analysis of gene connectivity in biological networks. Bioinformatics 31:442-444.

46. Li T, Wernersson R, Hansen RB, Horn H, Mercer J, Slodkowicz G, et al. (2017): A scored human protein-protein interaction network to catalyze genomic interpretation. Nat Methods 14:61-64.

47. Benjamini $Y$, Hochberg $Y$ (1995): Controlling the false discovery rate: A practical and powerful approach to multiple testing. J R Stat Soc Ser B 57:289-300.

48. Storey JD, Tibshirani R (2003): Statistical significance for genomewide studies. Proc Natl Acad Sci U S A 100:9440-9445.
49. Dudbridge F, Gusnanto A (2008): Estimation of significance thresholds for genomewide association scans. Genet Epidemiol 32:227-234.

50. Kambeitz J, Abi-Dargham A, Kapur S, Howes OD (2014): Alterations in cortical and extrastriatal subcortical dopamine function in schizophrenia: Systematic review and meta-analysis of imaging studies. $\mathrm{Br} \mathrm{J}$ Psychiatry 204:420-429.

51. Silva PN, Furuya TK, Braga IL, Rasmussen LT, Labio RW, Bertolucci PH, et al. (2014): Analysis of HSPA8 and HSPA9 mRNA expression and promoter methylation in the brain and blood of Alzheimer's disease patients. J Alzheimers Dis 38:165-170.

52. Anitha A, Nakamura K, Thanseem I, Yamada K, Iwayama Y, Toyota T, et al. (2012): Brain region-specific altered expression and association of mitochondria-related genes in autism. Mol Autism 3:12.

53. Murck H (2002): Magnesium and affective disorders. Nutritional neuroscience 5:375-389.

54. Kane JM (2015): The role of NMDA receptors in schizophrenia. J Clin Psychiatry 76:e1.

55. Machado-Vieira R, Zanetti MV, Teixeira AL, Uno M, Valiengo LL, Soeiro-de-Souza MG, et al. (2015): Decreased AKT1/mTOR pathway mRNA expression in short-term bipolar disorder. Eur Neuropsychopharmacol 25:468-473.

56. Vo MT, Ko MS, Lee UH, Yoon EH, Lee BJ, Cho WJ, et al. (2017): Developmentally regulated GTP-binding protein 2 depletion leads to mitochondrial dysfunction through downregulation of dynamin-related protein 1. Biochem Biophys Res Commun 486:1014-1020.

57. Lin YF, Schulz AM, Pellegrino MW, Lu Y, Shaham S, Haynes CM (2016): Maintenance and propagation of a deleterious mitochondrial genome by the mitochondrial unfolded protein response. Nature 533:416-419. 